\title{
Classical and Quantum Dynamics on Orbifolds
}

\author{
Yuri A. KORDYUKOV \\ Institute of Mathematics, Russian Academy of Sciences, \\ 112 Chernyshevsky Str., Ufa 450008, Russia \\ E-mail: yurikor@matem.anrb.ru
}

Received September 04, 2011, in final form November 20, 2011; Published online November 23, 2011 http://dx.doi.org/10.3842/SIGMA.2011.106

\begin{abstract}
We present two versions of the Egorov theorem for orbifolds. The first one is a straightforward extension of the classical theorem for smooth manifolds. The second one considers an orbifold as a singular manifold, the orbit space of a Lie group action, and deals with the corresponding objects in noncommutative geometry.
\end{abstract}

Key words: microlocal analysis; noncommutative geometry; symplectic reduction; quantization; foliation; orbifold; Hamiltonian dynamics; elliptic operators

2010 Mathematics Subject Classification: 58J40; 58J42; 58B34

\section{Introduction}

The Egorov theorem is a fundamental fact in microlocal analysis and mathematical physics. It relates the evolution of pseudodifferential operators on a compact manifold (quantum observables) determined by a first-order elliptic operator with the corresponding evolution of classical observables - the bicharacteristic flow on the space of symbols. This theorem is the rigorous version of the classical-quantum correspondence in quantum mechanics.

Let $P$ be an elliptic, first-order pseudodifferential operator on a compact manifold $X$ with real principal symbol $p \in S^{1}\left(T^{*} X\right)$. Let $f_{t}$ be the bicharacteristic flow of the operator $P$, that is, the Hamiltonian flow of $p$ on $T^{*} X$. The Egorov theorem states that, for any pseudodifferential operator $A$ of order 0 with the principal symbol $a \in S^{0}\left(T^{*} X\right)$, the operator $A(t)=e^{i t P} A e^{-i t P}$ is a pseudodifferential operator of order 0 . The principal symbol $a_{t} \in S^{0}\left(T^{*} X\right)$ of this operator is given by the formula

$$
a_{t}(x, \xi)=a\left(f_{t}(x, \xi)\right), \quad(x, \xi) \in T^{*} X \backslash\{0\} .
$$

Here $S^{m}\left(T^{*} X\right)$ denote the space of smooth functions on $T^{*} X \backslash\{0\}$, homogeneous of degree $m$ with respect to a fiberwise $\mathbb{R}$-action on $T^{*} X$.

The main purpose of this paper is to extend the Egorov theorem to orbifolds. We present two versions of the Egorov theorem. The first one is a straightforward extension of the classical theorem. The second one concerns with noncommutative geometry. It considers an orbifold as a singular manifold, the orbit space of a Lie group action, and deals with the corresponding noncommutative objects.

Spectral theory of elliptic operators on orbifolds has received much attention recently (see, for instance, a brief survey in the introduction of [3]). In [17], the Duistermaat-Guillemin trace formula was extended to compact Riemannian orbifolds. This formula has been applied in [6] to an inverse spectral problem on some orbifolds. We believe that our results will play an important role for the study of spectral asymptotics for elliptic operators on orbifolds, in particular, for the study of problems related to quantum ergodicity. 


\section{Classical theory}

\section{$2.1 \quad$ Orbifolds}

In this section we briefly review the basic definitions concerning orbifolds. For more details on orbifold theory we refer the reader to the book [1].

Let $X$ be a Hausdorff topological space. An $n$-dimensional orbifold chart on $X$ is given by a triple $\left(\tilde{U}, G_{U}, \phi_{U}\right)$, where $\tilde{U} \subset \mathbb{R}^{n}$ is a connected open subset, $G_{U}$ is a finite group acting on $\tilde{U}$ smoothly and $\phi_{U}: \tilde{U} \rightarrow X$ is a continuous map, which is $G_{U}$-invariant $\left(\phi_{U} \circ g=\phi_{U}\right.$ for all $g \in G_{U}$ ) and induces a homeomorphism of $\tilde{U} / G_{U}$ onto an open subset $U=\phi_{U}(\tilde{U}) \subset X$. An embedding $\lambda:\left(\tilde{U}, G_{U}, \phi_{U}\right) \rightarrow\left(\tilde{V}, G_{V}, \phi_{V}\right)$ between two such charts is a smooth embedding $\lambda: \tilde{U} \rightarrow \tilde{V}$ with $\phi_{V} \circ \lambda=\phi_{U}$.

An orbifold atlas on $X$ is a family $\mathcal{U}=\left\{\left(\tilde{U}, G_{U}, \phi_{U}\right)\right\}$ of orbifold charts, which cover $X$ and are locally compatible: given any two charts $\left(\tilde{U}, G_{U}, \phi_{U}\right)$ for $\phi_{U}(\tilde{U})=U \subset X$ and $\left(\tilde{U}, G_{V}, \phi_{V}\right)$ for $\phi_{V}(\tilde{V})=V \subset X$, and a point $x \in U \cap V$, there exists an open neighborhood $W$ of $x$ and a chart $\left(\tilde{W}, G_{W}, \phi_{W}\right)$ for $W$ such that there are embeddings $\lambda_{U}:\left(\tilde{W}, G_{W}, \phi_{W}\right) \hookrightarrow\left(\tilde{U}, G_{U}, \phi_{U}\right)$ and $\lambda_{V}:\left(\tilde{W}, G_{W}, \phi_{W}\right) \hookrightarrow\left(\tilde{V}, G_{V}, \phi_{V}\right)$.

An (effective) orbifold $X$ of dimension $n$ is a paracompact Hausdorff topological space equipped with an equivalence class of $n$-dimensional orbifold atlases.

Throughout in the paper, $X$ will denote a compact orbifold, $\operatorname{dim} X=n$.

Let $x \in X$ and $\left(\tilde{U}, G_{U}, \phi_{U}\right)$ be an orbifold chart such that $x \in U=\phi_{U}(\tilde{U})$. Take any $\tilde{x} \in \tilde{U}$ such that $\phi_{U}(\tilde{x})=x$. Let $G_{\tilde{x}} \subset G_{U}$ be the isotropy group for $\tilde{x}$. Up to conjugation, this group doesn't depend on the choice of chart and will be called the local group at $x$. A point $x \in X$ is called regular if the local group at $x$ is trivial and it is called singular otherwise. Denote by $X_{\text {reg }}$ the set of regular points of $X$ and by $X_{\text {sing }}$ the set of singular points of $X . X_{\text {reg }}$ is an open and dense subset of $X$ whose induced orbifold structure is a manifold structure.

A function $f: X \rightarrow \mathbb{C}$ is smooth iff for any orbifold chart $\left(\tilde{U}, G_{U}, \phi_{U}\right)$ the composition $\left.f\right|_{U} \circ \phi_{U}$ is a smooth function on $\tilde{U}$.

The cotangent bundle $T^{*} X$ of $X$ is an orbifold whose atlas is constructed as follows. Let $\left(\tilde{U}, G_{U}, \phi_{U}\right)$ is an orbifold chart over $U \subset X$. Consider the local cotangent bundle $T^{*} \tilde{U}=$ $\tilde{U} \times \mathbb{R}^{n}$. It is equipped with a natural action of the group $G_{U}$. The projection map $T^{*} \tilde{U} \rightarrow \tilde{U}$ is $G_{U}$-equivariant, so we obtain a natural projection $p_{U}: T^{*} \tilde{U} / G_{U} \rightarrow U$, whose fiber $p_{U}^{-1}(x)$ is homeomorphic to $\mathbb{R}^{n} / G_{\tilde{x}}$. $T^{*} X$ is obtained by gluing together the bundles $T^{*} \tilde{U} / G_{U}$ over each chart $U$ in the atlas of $X$. Namely, let $\left(\tilde{U}, G_{U}, \phi_{U}\right)$ and $\left(\tilde{V}, G_{V}, \phi_{V}\right)$ be two orbifold charts for $\phi_{U}(\tilde{U})=U \subset X$ and $\phi_{V}(\tilde{V})=V \subset X$ respectively, and let $x$ belong to $U \cap V$. There exists an open neighborhood $W$ of $x$ and a chart $\left(\tilde{W}, G_{W}, \phi_{W}\right)$ for $W$ such that there are embeddings $\lambda_{U}:\left(\tilde{W}, G_{W}, \phi_{W}\right) \hookrightarrow\left(\tilde{U}, G_{U}, \phi_{U}\right)$ and $\lambda_{V}:\left(\tilde{W}, G_{W}, \phi_{W}\right) \hookrightarrow\left(\tilde{V}, G_{V}, \phi_{V}\right)$. These embeddings give rise to diffeomorphisms $\lambda_{U}: \tilde{W} \rightarrow \lambda_{U}(\tilde{W}) \subset \tilde{U}$ and $\lambda_{V}: \tilde{W} \rightarrow \lambda_{V}(\tilde{W}) \subset \tilde{V}$, which provide an equivariant diffeomorphism $\lambda_{U V}=\lambda_{V} \lambda_{U}^{-1}: \lambda_{U}(\tilde{W}) \rightarrow \lambda_{V}(\tilde{W})$, the transition function. Then the bundles $p_{U}: T^{*} \tilde{U} / G_{U} \rightarrow U$ and $p_{V}: T^{*} \tilde{V} / G_{V} \rightarrow V$ are glued by the cotangent map $T^{*} \lambda_{V U}: T^{*} \lambda_{U}\left(T^{*} \tilde{W}\right) \rightarrow T^{*} \lambda_{V}\left(T^{*} \tilde{W}\right)$.

Like in the manifold case, the cotangent bundle $T^{*} X$ carries a canonical symplectic structure. Here by a symplectic form on an orbifold $Y$ we will understand an orbifold atlas $\mathcal{U}=$ $\left\{\left(\tilde{U}, G_{U}, \phi_{U}\right)\right\}$ together with a $G_{U}$-invariant symplectic form $\omega_{U}$ on $\tilde{U}$ for each $\left(\tilde{U}, G_{U}, \phi_{U}\right) \in \mathcal{U}$ such that, for any transition function $\lambda_{U V}: \lambda_{U}(\tilde{W}) \subset \tilde{U} \rightarrow \lambda_{V}(\tilde{W}) \subset \tilde{V}$ as above, we have $\lambda_{U V}^{*} \omega_{V}=\omega_{U}$. An orbifold $Y$ equipped with a symplectic form $\omega$ is called a symplectic orbifold. The symplectic structure on $T^{*} X$ can be constructed as follows. Consider the orbifold chart $\left(T^{*} \tilde{U}, G_{U}, T^{*} \phi_{U}\right)$ induced by an orbifold chart $\left(\tilde{U}, G_{U}, \phi_{U}\right)$. Then $T^{*} \tilde{U}$ carries a canonical symplectic form $\omega_{T^{*} \tilde{U}}$, which is invariant with respect to the lifted $G_{U^{-}}$action. These symplectic forms are compatible for two different orbifold charts and define a symplectic form on $T^{*} X$. 
The flow $F_{t}$ on a symplectic orbifold $(Y, \omega)$ is Hamiltonian with a Hamiltonian $H \in C^{\infty}(Y)$ if, in any orbifold chart $\left(\tilde{U}, G_{U}, \phi_{U}\right)$, the infinitesimal generator $X_{H} \in \mathcal{X}(\tilde{U})$ of the flow satisfies a standard relation

$$
i\left(X_{H}\right) \omega_{U}=d\left(\left.H\right|_{U} \circ \phi_{U}\right)
$$

Since $Y$ is not a manifold, this equation can not be reduced to a system of first-order ordinary differential equations on a manifold. Nevertheless, one can show the existence and uniqueness of the Hamiltonian flow with an arbitrary Hamiltonian $H$ (for instance, using quotient presentations, see below). The flow $F_{t}$ leaves the singular set of the orbifold $Y$ invariant, and its restriction to the regular part $Y_{\text {reg }}$ of $Y$ is the Hamiltonian flow of the function $\left.H\right|_{Y_{\text {reg }}}$ in the usual sense. We refer the reader to [16] for more information on Hamiltonian dynamics on singular symplectic spaces.

\subsection{Pseudodifferential operators on orbifolds}

Here we recall basic facts about pseudodifferential operators on orbifolds (see [2, 4, 5] for details). We start with some information about orbibundles.

A (real) vector orbibundle over an orbifold $X$ is given by an orbifold $E$ and a surjective continuous map $p: E \rightarrow X$ such that, for any $x_{0} \in X$, there exists an orbifold chart $\left(\tilde{U}, G_{U}, \phi_{U}\right)$ over $\phi_{U}(\tilde{U})=U \subset X$ with $x_{0} \in U$ and an orbifold chart $\left(\tilde{U} \times \mathbb{R}^{k}, G_{U}, \tilde{\phi}_{U}\right)$ over $\tilde{\phi}_{U}\left(\tilde{U} \times \mathbb{R}^{k}\right)=$ $p^{-1}(U) \subset E$ (called a local trivialization of $E$ over $\left.\left(\tilde{U}, G_{U}, \phi_{U}\right)\right)$ such that:

1) the action of $G_{U}$ on $\tilde{U} \times \mathbb{R}^{k}$ is an extension of the action of $G_{U}$ on $\tilde{U}$ given by

$$
g(x, v)=(g x, \rho(x, g) v), \quad x \in \tilde{U}, \quad v \in \mathbb{R}^{k},
$$

where $\rho$ is a smooth map from $\tilde{U} \times G_{U}$ to the algebra $\mathcal{L}\left(\mathbb{R}^{k}\right)$ of linear maps in $\mathbb{R}^{k}$ satisfying

$$
\rho(g x, h) \circ \rho(x, g)=\rho(x, h g), \quad g, h \in G_{U}, \quad x \in \tilde{U}
$$

(in other words, $G_{U}$ acts by vector bundle isomorphisms of the trivial vector bundle $\mathrm{pr}_{1}$ : $\left.\tilde{U} \times \mathbb{R}^{k} \rightarrow \tilde{U}\right)$

2) the map $\operatorname{pr}_{1}: \tilde{U} \times \mathbb{R}^{k} \rightarrow \tilde{U}$ satisfies $\phi_{U} \circ \operatorname{pr}_{1}=p \circ \tilde{\phi}_{U}$.

Moreover, any two local trivializations are compatible in a natural way.

The tangent bundle and the cotangent bundle of an orbifold $X$ are examples of real vector orbibundles over $X$.

A section $s: X \rightarrow E$ is called $C^{\infty}$, if for each local trivialization $\left(\tilde{U} \times \mathbb{R}^{k}, G_{U}, \tilde{\phi}_{U}\right)$ over an orbifold chart $\left(\tilde{U}, G_{U}, \phi_{U}\right)$ the restriction $\left.s\right|_{U}$ is covered by a $G_{U}$-invariant smooth section $\tilde{s}_{U}: \tilde{U} \rightarrow \tilde{U} \times \mathbb{R}^{k}:\left.s\right|_{U} \circ \phi_{U}=\tilde{\phi}_{U} \circ \tilde{s}_{U}$. We denote by $C^{\infty}(X, E)$ the space of smooth section of $E$ on $X$.

Now we turn to pseudodifferential operators. Let $X$ be a compact orbifold, and $E$ a complex vector orbibundle over $X$. A linear mapping $P: C^{\infty}(X, E) \rightarrow C^{\infty}(X, E)$ is a (pseudo) differential operator on $X$ of order $m$ iff:

(1) the Schwartz kernel of $P$ is smooth outside of a neighborhood of the diagonal in $X \times X$.

(2) for any $x_{0} \in X$ and for any local trivialization $\left(\tilde{U} \times \mathbb{C}^{k}, G_{U}, \tilde{\phi}_{U}\right)$ of $E$ over an orbifold chart $\left(\tilde{U}, G_{U}, \phi_{U}\right)$ with $x_{0} \in U$, the operator $\left.C_{c}^{\infty}\left(U,\left.E\right|_{U}\right) \ni f \mapsto P(f)\right|_{U} \in C^{\infty}\left(U,\left.E\right|_{U}\right)$ is given by the restriction to $G_{U^{-}}$invariant functions of a (pseudo)differential operator $\tilde{P}$ of order $m$ on $C^{\infty}\left(\tilde{U}, \mathbb{C}^{k}\right)$ that commutes with the induced $G_{U}$ action on $C^{\infty}\left(\tilde{U}, \mathbb{C}^{k}\right)$. 
All our pseudodifferential operators are assumed to be classical (or polyhomogeneous), that is, their complete symbols can be represented as an asymptotic sum of homogeneous components. Denote by $\Psi^{m}(X, E)$ the class of pseudodifferential operators of order $m$ acting on $C^{\infty}(X, E)$.

It is not hard to show [2, Proposition 3.3] that the operator $\tilde{P}$ is unique up to a smoothing operator, so it is unique if $P$ is a differential operator. A pseudodifferential operator $\tilde{P}$ on $\tilde{U}$ that commutes with the action of $G_{U}$ has a principal symbol $\sigma(\tilde{P}) \in C^{\infty}\left(\tilde{U} \times\left(\mathbb{R}^{n} \backslash\{0\}\right), \mathcal{L}\left(\mathbb{C}^{k}\right)\right)$ that is invariant with respect to the natural $G_{U}$-action. One can check that these locally defined functions determine a global smooth section $\sigma(P)$ of the vector orbibundle $\operatorname{End}\left(\pi^{*} E\right)$ on $T^{*} X \backslash\{0\}$, the principal symbol of $P$. (Here $\pi: T^{*} X \rightarrow X$ is the bundle map and $\pi^{*} E$ is the pull-back of the orbibundle $E$ under the map $\pi$.) The pseudodifferential operator $P$ on $X$ is elliptic if $\tilde{P}$ is elliptic for all choices of orbifold charts.

\subsection{Classical version of the Egorov theorem}

Let $X$ be a compact orbifold, and $P$ an elliptic, first-order pseudodifferential operator on $X$ with real principal symbol $p \in S^{1}\left(T^{*} X\right)$. Let $f_{t}$ be the bicharacteristic flow of the operator $P$, that is, the Hamiltonian flow of $p$ on the cotangent bundle $T^{*} X$.

As an example, one can consider $P=\sqrt{\Delta_{X}}$, where $\Delta_{X}$ is the Laplace-Beltrami operator associated to a Riemannian metric $g_{X}$ on $X$. Its bicharacteristic flow is the geodesic flow of the metric $g_{X}$ on $T^{*} X$.

The classical version of the Egorov theorem for orbifolds reads as follows.

Theorem 1. For any pseudodifferential operator $A$ of order 0 with the principal symbol $a \in$ $S^{0}\left(T^{*} X\right)$, the operator

$$
A(t)=e^{i t P} A e^{-i t P}
$$

is a pseudodifferential operator of order 0. Moreover, its principal symbol $a_{t} \in S^{0}\left(T^{*} X\right)$ is given by

$$
a_{t}(x, \xi)=a\left(f_{t}(x, \xi)\right), \quad(x, \xi) \in T^{*} X \backslash 0 .
$$

The proof of Theorem 1 will be given in Section 2.8 .

Remark 1. The classical Egorov theorem plays a crucial role in the proof of the well-known result due to Shnirelman, stating that the ergodicity of the bicharacteristic flow of a first-order elliptic pseudodifferential operator on a compact manifold implies quantum ergodicity for the operator itself. We will discuss these issues for orbifolds elsewhere.

\subsection{The Egorov theorem for matrix-valued operators}

Using the results of [7], one can easily extend Theorem 1 to pseudodifferential operators acting on sections of a vector orbibundle over a compact orbifold.

Let $X$ be a compact orbifold, $E$ a complex vector orbibundle on $X$, and $P$ an elliptic, first-order pseudodifferential operator acting on $C^{\infty}\left(X,|T X|^{1 / 2} \otimes E\right)$ with real scalar principal symbol $p_{1} \in S^{1}\left(T^{*} X, \operatorname{End}\left(\pi^{*} E\right)\right), p_{1}(x, \xi)=h(x, \xi) \operatorname{id}_{E_{x}}$ with $h \in C^{\infty}\left(T^{*} X \backslash\{0\}\right)$. (Here $|T X|^{1 / 2}$ denotes the half-density line orbibundle on $X$.) Let $H_{h}$ be the associated Hamiltonian vector field and $f_{t}$ the associated Hamiltonian flow on $T^{*} X$.

Consider a local trivialization $\left(\tilde{U} \times \mathbb{C}^{k}, G_{U}, \tilde{\phi}_{U}\right)$ over an orbifold chart $\left(\tilde{U}, G_{U}, \phi_{U}\right)$. Let $\tilde{P}$ be the corresponding $G_{U}$-invariant, matrix-valued first-order pseudodifferential operator on $\tilde{U}$. The 
subprincipal symbol of $\tilde{P}$ is a smooth matrix-valued function $\operatorname{sub}(\tilde{P}) \in C^{\infty}\left(\tilde{U} \times\left(\mathbb{R}^{n} \backslash\{0\}\right), \mathcal{L}\left(\mathbb{C}^{k}\right)\right)$ defined by

$$
\operatorname{sub}(\tilde{P})(x, \xi)=p_{0}(x, \xi)-\frac{1}{2 i} \sum_{j=1}^{n} \frac{\partial^{2} p_{1}}{\partial x_{j} \partial \xi_{j}}(x, \xi), \quad x \in \tilde{U}, \quad \xi \in \mathbb{R}^{n} \backslash\{0\}
$$

where $p_{k}$ is the homogeneous of degree $k$ component in the asymptotic expansion of the complete symbol of $\tilde{P}$.

If $E$ is trivial, the subprincipal symbol turns out to be well defined as a function on $T^{*} X$. In the general case, we consider the first-order differential operator

$$
\nabla_{H_{h}}:=H_{h}+i \operatorname{sub}(\tilde{P})
$$

acting on $C^{\infty}\left(\tilde{U} \times\left(\mathbb{R}^{n} \backslash\{0\}\right), \mathbb{C}^{k}\right)$. By [7], the operator $\nabla_{H_{h}}$ is invariantly defined as a covariant derivative (a partial connection) on the vector orbibundle $\pi^{*} E$ on $T^{*} X \backslash\{0\}$ along the Hamiltonian vector field $H_{h}$.

This determines a flow $\alpha_{t}$ on $\pi^{*} E$ by

$$
\alpha_{t}(x, \xi, v)=(x(t), \xi(t), v(t)), \quad(x, \xi) \in T^{*} X \backslash\{0\}, \quad v \in\left(\pi^{*} E\right)_{(x, \xi)} \cong E_{x},
$$

where $(x(t), \xi(t))=f_{t}(x, \xi)$ and, in local coordinates, $v(t)$ satisfies

$$
\frac{d v(t)}{d t}=i \operatorname{sub}(\tilde{P})(x(t), \xi(t)) v(t)
$$

The induced flow $\alpha_{t}^{*}$ on $C^{\infty}\left(T^{*} X \backslash\{0\}, \pi^{*} E\right)$ satisfies

$$
\frac{d}{d t} \alpha_{t}^{*} f=\nabla_{H_{h}} f
$$

There is also a flow $\operatorname{Ad}\left(\alpha_{t}\right)$ on $\operatorname{End}\left(\pi^{*} E\right)$, which, in its turn, induces a flow $\operatorname{Ad}\left(\alpha_{t}\right)^{*}$ on the space $C^{\infty}\left(T^{*} X \backslash\{0\}, \operatorname{End}\left(\pi^{*} E\right)\right)$. If $f \in C^{\infty}\left(T^{*} X \backslash\{0\}, \operatorname{End}\left(\pi^{*} E\right)\right)$,

$$
\frac{d}{d t} \operatorname{Ad}\left(\alpha_{t}\right)^{*} f=\left[\nabla_{H_{h}}, f\right]
$$

Theorem 2. For any $A \in \Psi^{0}(X, E)$ with the principal symbol $a \in S^{0}\left(T^{*} X, \operatorname{End}\left(\pi^{*} E\right)\right)$, the operator

$$
A(t)=e^{i t P} A e^{-i t P}
$$

is a pseudodifferential operator of order 0 . Moreover, its principal symbol $a_{t} \in S^{0}\left(T^{*} X, \operatorname{End}\left(\pi^{*} E\right)\right)$ is given by

$$
a_{t}=\operatorname{Ad}\left(\alpha_{t}\right)^{*} a
$$

\subsection{Quotient presentations}

We will need the following well-known fact from orbifold theory due to Kawasaki [8, 9] (see, for instance, $[2,15]$ for a detailed proof).

Proposition 1. Let $M$ be a smooth manifold and $K$ a compact Lie group acting on $M$ with finite isotropy groups. Then the quotient $X=M / K$ (with the quotient topology) has a natural orbifold structure. Conversely, any orbifold is a quotient of this type. 
Any representation of an orbifold $X$ as the quotient $X \cong M / K$ of an action of a compact Lie group $K$ on a smooth manifold $M$ with finite isotropy groups will be called a quotient presentation for $X$. There is a classical example of a quotient presentation for an orbifold $X$ due to Satake. Choose a Riemannian metric on $X$. It can be shown that the orthonormal frame bundle $M=F(X)$ of the Riemannian orbifold $X$ is a smooth manifold, the group $K=O(n)$ acts smoothly, effectively and locally freely on $M$, and $M / K \cong X$.

Remark 2. More generally, one can consider realizations of an orbifold as the leaf space of a foliated manifold with all leaves compact and all holonomy groups finite (a generalized Seifert fibration). The holonomy groupoid of such a foliation is a proper effective groupoid, which provides a characterization of orbifolds in terms of groupoids.

Note that if $X \cong M / K$ is a quotient presentation for $X$, then the pull-back by the natural projection $M \rightarrow X$ is an isomorphism $C^{\infty}(X) \cong C^{\infty}(M)^{K}$ between the smooth functions on $X$ and the $K$-invariant functions on $M$.

There is the following extension of Proposition 1 observed by Kawasaki (see, for instance, [2] for a detailed proof).

Proposition 2. Let $\mathcal{E}$ be a smooth vector bundle over a smooth manifold $M$ and $K$ a compact Lie group acting on $\mathcal{E}$ by vector bundle isomorphisms such that isotropy groups on $M$ are finite. Then the quotient map $E=\mathcal{E} / K \rightarrow X=M / K$ has a canonical structure of a vector orbibundle. Conversely, any vector orbibundle is a quotient of this type.

Moreover (see, for instance, [2, Proposition 2.4]), if $X \cong M / K$ is a quotient presentation for $X, E$ is a vector orbibundle on $X$, and $\mathcal{E}$ is the smooth vector bundle over $M$ given by Proposition 2, then

$$
C^{\infty}(M, \mathcal{E})^{K} \cong C^{\infty}(X, E) .
$$

\subsection{Quotient presentations of the cotangent bundle}

A quotient presentation $X \cong M / K$ for the orbifold $X$ gives rise to a quotient presentation for the cotangent bundle $T^{*} X$ of $X$ in the following way. The action of $K$ on $M$ induces an action of $K$ on the cotangent bundle $T^{*} M$. Denote by $\mathfrak{k}$ the Lie algebra of $K$. For any $v \in \mathfrak{k}$, denote by $v_{M}$ the corresponding infinitesimal generator of the $K$-action on $M$. For any $x \in M$, vectors of the form $v_{M}(x)$ with $v \in \mathfrak{k}$ span the tangent space $T_{x}(K x)$ to the $K$-orbit, passing through $x$. Denote

$$
\left(T_{K}^{*} M\right)_{x}=\left\{\xi \in T_{x}^{*} M:\left\langle\xi, v_{M}(x)\right\rangle=0 \text { for any } v \in \mathfrak{k}\right\} .
$$

Since the action is locally free, the disjoint union

$$
T_{K}^{*} M=\bigsqcup_{x \in M}\left(T_{K}^{*} M\right)_{x}
$$

is a subbundle of the cotangent bundle $T^{*} M$, called the conormal bundle. The conormal bundle $T_{K}^{*} M$ is a $K$-invariant submanifold of $T^{*} M$ such that

$$
T_{K}^{*} M / K \cong T^{*} X .
$$

This gives a quotient presentation for $T^{*} X$.

This construction is a particular case of the symplectic reduction. Indeed, the $K$-action on $T^{*} M$ is a Hamiltonian action with the corresponding momentum map $J: T^{*} M \rightarrow \mathfrak{k}^{*}$ given by

$$
\langle J(x, \xi), v\rangle=\left\langle\xi, v_{M}(x)\right\rangle, \quad(x, \xi) \in T^{*} M, \quad v \in \mathfrak{k} .
$$


Thus, we see that

$$
T_{K}^{*} M=J^{-1}(0),
$$

and the quotient $T_{K}^{*} M / K$ is the Marsden-Weinstein reduced space $M_{0}$ at $0 \in \mathfrak{k}^{*}$ [14].

Using quotient presentations, one can show the existence of Hamiltonian flows on $T^{*} X$. Let $X \cong M / K$ be a quotient presentation for $X$. Consider a Hamiltonian $H \in C^{\infty}\left(T^{*} X\right)$ as a smooth $K$-invariant function on $T_{K}^{*} M$. Let $\tilde{H} \in C^{\infty}\left(T^{*} M\right)^{K}$ be an arbitrary extension of $H$ to a smooth $K$-invariant function on $T^{*} M$. Let $\tilde{f}_{t}$ be the Hamiltonian flow of $\tilde{H}$ on $T^{*} M$. Since $\tilde{H}$ is $K$-invariant, the flow $\tilde{f}_{t}$ preserves the conormal bundle $T_{K}^{*} M$, and its restriction to $T_{K}^{*} M$ (denoted also by $\tilde{f}_{t}$ ) commutes with the $K$-action on $T_{K}^{*} M$. So the flow $\tilde{f}_{t}$ on $T_{K}^{*} M$ induces a flow $f_{t}$ on the quotient $T_{K}^{*} M / K=T^{*} X$, which is called the reduced flow. One can show that this flow is a Hamiltonian flow on $T^{*} X$ with Hamiltonian $H$.

\subsection{Pseudodifferential operators and quotient presentations}

Let $X$ be a compact orbifold and $E$ a complex vector orbibundle on $X$. Let $X \cong M / K$ be a quotient presentation for $X$ and let $\mathcal{E}$ be the lift of $E$ to a smooth vector bundle over $M$ given by Proposition 2. Let us consider $C^{\infty}(X, E)\left(\right.$ resp. $\left.L^{2}(X, E)\right)$ as a subspace $C^{\infty}(M, \mathcal{E})^{K}$ (resp. $\left.L^{2}(M, \mathcal{E})^{K}\right)$ of $C^{\infty}(M, \mathcal{E})$ (resp. $L^{2}(M, \mathcal{E})$ ), which consists of $K$-invariant functions on $M$. Let $\Pi: L^{2}(M, \mathcal{E}) \rightarrow L^{2}(M, \mathcal{E})^{K} \cong L^{2}(X, E)$ be the orthogonal projection onto $L^{2}(M, \mathcal{E})^{K}$ in $L^{2}(M, \mathcal{E})$. It is clear that $\Pi\left(C^{\infty}(M, \mathcal{E})\right)=C^{\infty}(M, \mathcal{E})^{K} \cong C^{\infty}(X, E)$.

For any pseudodifferential operator $B \in \Psi^{m}(M, \mathcal{E})$, define its transversal principal symbol $\sigma(B) \in S^{m}\left(T_{K}^{*} M, \operatorname{End}\left(\tilde{\pi}^{*} \mathcal{E}\right)\right)$, where $\tilde{\pi}: T^{*} M \backslash\{0\} \rightarrow M$ is the bundle map, as the restriction of the principal symbol of $B$ to $T_{K}^{*} M$. If $B$ is $K$-invariant, then $\sigma(B)$ is $K$-invariant, so it can be identified with an element of the space $S^{m}\left(T^{*} X, \operatorname{End}\left(\pi^{*} E\right)\right)$.

We have the following fact, relating pseudodifferential operators on $M$ and $X$ (see [2, Proposition 3.4] and [17, Proposition 2.3]).

Proposition 3. Given a linear operator $A: C^{\infty}(X, E) \rightarrow C^{\infty}(X, E), A$ is a pseudodifferential operator on $X$ iff there exists a pseudodifferential operator $\tilde{A}: C^{\infty}(M, \mathcal{E}) \rightarrow C^{\infty}(M, \mathcal{E})$ (of the same order as $A)$ which commutes with $K$, such that $\Pi \tilde{A} \Pi=A$.

The transverse principal symbol $\sigma(\tilde{A}) \in S^{0}\left(T_{K}^{*} M, \operatorname{End}\left(\tilde{\pi}^{*} \mathcal{E}\right)\right)^{K}$ of $\tilde{A}$ coincides with the principal symbol $\sigma(A) \in S^{0}\left(T^{*} X, \operatorname{End}\left(\pi^{*} E\right)\right)$ of $A$ under the identification $C^{\infty}\left(T_{K}^{*} M, \operatorname{End}\left(\tilde{\pi}^{*} \mathcal{E}\right)\right)^{K} \cong$ $C^{\infty}\left(T^{*} X, \operatorname{End}\left(\pi^{*} E\right)\right)$.

Now we are ready to complete the proofs of Theorems 1 and 2.

\subsection{Proofs of Theorems 1 and 2}

Let $P \in \Psi^{1}(X)$ be an elliptic operator on $X$ with real principal symbol $p \in S^{1}\left(T^{*} X\right)$ and $A \in \Psi^{0}(X)$. Let $X \cong M / K$ be a quotient presentation for $X$. Take $\tilde{P} \in \Psi^{1}(M)$ and $\tilde{A} \in \Psi^{0}(M)$ as in Proposition 3. Without loss of generality, we can also assume that $\tilde{P}$ is elliptic and its principal symbol is real. So we have

$$
\Pi \tilde{P} \Pi=P, \quad \Pi \tilde{A} \Pi=A .
$$

Since $\tilde{P}$ is $K$-invariant, we have $\tilde{P} \Pi=\Pi \tilde{P}$. Using these facts, we easily derive that

$$
e^{i t P} A e^{-i t P}=\Pi e^{i t \tilde{P}} \tilde{A} e^{-i t \tilde{P}} \Pi \text {. }
$$

By the classical Egorov theorem, the operator $e^{i t \tilde{P}} \tilde{A} e^{-i t \tilde{P}}$ is in $\Psi^{0}(M)$. Since it commutes with the $K$-action, we conclude that $e^{i t P} A e^{-i t P} \in \Psi^{0}(X)$. Moreover, for the transversal principal 
symbol of $e^{i t \tilde{P}} \tilde{A} e^{-i t \tilde{P}}$, we have

$$
\sigma\left(e^{i t \tilde{P}} \tilde{A} e^{-i t \tilde{P}}\right)(\nu)=\sigma(\tilde{A})\left(\tilde{f}_{t}(\nu)\right), \quad \nu \in T_{K}^{*} M \backslash\{0\},
$$

where $\tilde{f}_{t}$ is the Hamiltonian flow of $\tilde{p}$, the principal symbol of $\tilde{P}$.

By Proposition 3, we have

$$
\sigma\left(e^{i t \tilde{P}} \tilde{A} e^{-i t \tilde{P}}\right)(\nu)=\sigma\left(e^{i t P} A e^{-i t P}\right)(\nu), \quad \nu \in T_{K}^{*} M / K \backslash\{0\} \cong T^{*} X \backslash\{0\},
$$

that immediately completes the proof of Theorem 1.

The proof of Theorem 2 is similar. Under the assumptions of Theorem 2 , let $X \cong M / K$ be a quotient presentation for $X$ and $\mathcal{E}$ is the lift of $E$ to a $K$-equivariant smooth vector bundle on $M$ given by Proposition 2. Take $\tilde{P} \in \Psi^{1}(M, \mathcal{E})$ and $\tilde{A} \in \Psi^{0}(M, \mathcal{E})$ as in Proposition 3. Without loss of generality, we can assume that $\tilde{P}$ is elliptic and its principal symbol is scalar and real. Let $\tilde{f}_{t}$ be the Hamiltonian flow of $\tilde{h} \in C^{\infty}\left(T^{*} M \backslash\{0\}\right)$, the principal symbol of $\tilde{P}$. The subprincipal symbol of $\tilde{P}$ is invariantly defined as a partial connection $\nabla_{H_{\tilde{h}}}$ on the vector orbibundle $\tilde{\pi}^{*} \mathcal{E}$ along the Hamiltonian vector field $H_{\tilde{h}}$. Therefore, we have the flow $\tilde{\alpha}_{t}^{*}$ on $C^{\infty}\left(T^{*} M \backslash\{0\}, \operatorname{End}\left(\tilde{\pi}^{*} \mathcal{E}\right)\right)$, which satisfies

$$
\frac{d}{d t} \tilde{\alpha}_{t}^{*} f=\nabla_{H_{\tilde{h}}} f, \quad f \in C^{\infty}\left(T^{*} M \backslash\{0\}, \operatorname{End}\left(\tilde{\pi}^{*} \mathcal{E}\right)\right),
$$

and the flow $\operatorname{Ad}\left(\tilde{\alpha}_{t}\right)^{*}$ on $C^{\infty}\left(T^{*} M \backslash\{0\}, \operatorname{End}\left(\tilde{\pi}^{*} \mathcal{E}\right)\right)$, which satisfies

$$
\frac{d}{d t} \operatorname{Ad}\left(\tilde{\alpha}_{t}\right)^{*} f=\left[\nabla_{H_{\tilde{h}}}, f\right], \quad f \in C^{\infty}\left(T^{*} M \backslash\{0\}, \operatorname{End}\left(\tilde{\pi}^{*} \mathcal{E}\right)\right) .
$$

By $K$-invariance, the restriction of $\tilde{\alpha}_{t}^{*}$ to $C^{\infty}\left(T_{K}^{*} M \backslash\{0\}, \tilde{\pi}^{*} \mathcal{E}\right)$ takes $C^{\infty}\left(T_{K}^{*} M \backslash\{0\}, \tilde{\pi}^{*} \mathcal{E}\right)^{K}$ to itself, and there is the following commutative diagram:

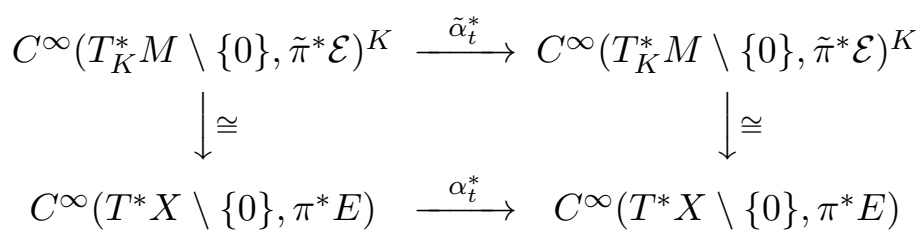

A similar statement holds for $\operatorname{Ad}\left(\tilde{\alpha}_{t}\right)^{*}$. Taking into account these facts, Theorem 2 is a direct consequence of Egorov's theorem in [7].

\section{Noncommutative geometry}

\subsection{The operator algebras associated with a quotient orbifold}

Let $X$ be a compact orbifold. The choice of quotient presentation $X \cong M / K$ for $X$ allows us to consider $X$ as the orbit space of a Lie group action, which is a typical object of noncommutative geometry. So we can use some notions and ideas of noncommutative geometry.

First, one can consider the smooth crossed product algebra $C^{\infty}(M) \rtimes K$. As a linear space, $C^{\infty}(M) \rtimes K=C^{\infty}(M \times K)$. The product in $C^{\infty}(M \times K)$ is given, for any functions $f, g \in$ $C^{\infty}(M \times K)$, by

$$
(f * g)(x, k)=\int_{K} f(x, h) g\left(h^{-1} x, h^{-1} k\right) d h, \quad(x, k) \in M \times K,
$$

the involution is given, for a function $f \in C^{\infty}(M \times K)$, by

$$
f^{*}(x, k)=\overline{f\left(k^{-1} x, k^{-1}\right)}, \quad(x, k) \in M \times K .
$$

Here $d h$ denotes a fixed bi-invariant Haar measure on $K$. 
It is useful to know that the crossed product algebra $C^{\infty}(M) \rtimes K$ is associated with a certain groupoid, the transformation groupoid, $G=M \rtimes K$. As a set, $G=M \times K$. It is equipped with the source map $s: M \times K \rightarrow M$ given by $s(x, k)=k^{-1} x$ and the target map $r: M \times K \rightarrow M$ given by $r(x, k)=x$.

For any $x \in M$, there is a natural $*$-representation of the algebra $C^{\infty}(M \times K)$ in the Hilbert space $L^{2}(K, d k)$ given, for $f \in C^{\infty}(M \times K)$ and $\zeta \in L^{2}(K, d k)$, by

$$
R_{x}(f) \zeta(k)=\int_{K} f\left(k^{-1} x, k^{-1} k_{1}\right) \zeta\left(k_{1}\right) d k_{1} .
$$

The completion of the involutive algebra $C^{\infty}(M \times K)$ in the norm

$$
\|f\|=\sup _{x \in M}\left\|R_{x}(f)\right\|
$$

is called the reduced crossed product $C^{*}$-algebra and denoted by $C(M) \rtimes_{r} K$.

Since $K$ is compact, this algebra coincides with the full crossed product $C^{*}$-algebra $C(M) \rtimes K$, which is defined as the completion of $C^{\infty}(M \times K)$ in the norm

$$
\|k\|_{\max }=\sup \|\pi(k)\|,
$$

where supremum is taken over the set of all $*$-representations $\pi$ of the algebra $C^{\infty}(M \times K)$ in Hilbert spaces.

There is also a natural representation of $C^{\infty}(M \times K)$ in $L^{2}(M)$ defined for $f \in C^{\infty}(M \times K)$ and $u \in L^{2}(M)$ by

$$
R(f) u(x)=\int_{K} f(x, k) u\left(k^{-1} x\right) d k, \quad x \in M .
$$

This representation extends to a $*$-representation of $C(M) \rtimes_{r} K$.

The $C^{*}$-algebra $C(M) \rtimes_{r} K$ can be naturally called the orbifold $C^{*}$-algebra associated to the quotient presentation $X \cong M / K$. From the point of view of noncommutative geometry, this algebra is a noncommutative analogue of the algebra of continuous functions on the quotient space $M / K$. It is Morita equivalent to the commutative algebra $C(X)$.

\subsection{Noncommutative pseudodifferential operators on orbifolds}

Let $X$ be a compact orbifold. One can introduce the algebra $\Psi^{*}(M / K)$ of noncommutative pseudodifferential operators on $X$ associated with a quotient presentation $X \cong M / K$.

First, let us start with a local definition. Constructing an appropriate slice for the $K$-action on $M$, one can give the following local description of the quotient map $p: M \rightarrow X$ (see, for instance, [2, Proposition 2.1] for details).

Proposition 4. For any $x \in X$, there exists an orbifold chart $\left(\tilde{U}, G_{U}, \phi_{U}\right)$ defined in a neighborhood $U \subset X$ of $x$ such that there exists a $K$-equivariant diffeomorphism

$$
p^{-1}(U) \cong K \times_{G_{U}} \tilde{U}
$$

Recall that, by definition, $K \times{ }_{G_{U}} \tilde{U}=(K \times \tilde{U}) / G_{U}$, where $G_{U}$ acts on $K \times \tilde{U}$ by

$$
\gamma \cdot(k, y)=\left(k \gamma^{-1}, \gamma y\right), \quad k \in K, \quad y \in \tilde{U}, \quad \gamma \in G_{U}
$$

and the $K$-action on $K \times_{G_{U}} \tilde{U}$ is given by the left translations on $K$. 
Now consider an orbifold chart $\left(\tilde{U}, G_{U}, \phi_{U}\right)$ as in Proposition 4 . For any $a \in S^{m}(K \times K \times$ $\left.\tilde{U} \times \mathbb{R}^{n}\right)$, define an operator

$$
\bar{A}: C_{c}^{\infty}(K \times \tilde{U}) \rightarrow C^{\infty}(K \times \tilde{U})
$$

by the formula

$$
\bar{A} u(k, y)=(2 \pi)^{-n} \int e^{i\left(y-y^{\prime}\right) \eta} a\left(k, k^{\prime}, y, \eta\right) u\left(k^{\prime}, y^{\prime}\right) d k^{\prime} d y^{\prime} d \eta
$$

where $u \in C_{c}^{\infty}(K \times \tilde{U}), k \in K, y \in \tilde{U}$.

Assume that the operator $\bar{A}$ commutes with the action of $G_{U}$ on $K \times \tilde{U}$. Then $\bar{A}$ defines an operator

$$
A: C_{c}^{\infty}\left(K \times_{G_{U}} \tilde{U}\right) \cong C_{c}^{\infty}\left(p^{-1}(U)\right) \rightarrow C^{\infty}\left(K \times_{G_{U}} \tilde{U}\right) \cong C^{\infty}\left(p^{-1}(U)\right) .
$$

If, in addition, the Schwartz kernel of the operator $\bar{A}$ is compactly supported in $(K \times \tilde{U}) \times(K \times \tilde{U})$, then the operator $A$ acts from $C_{c}^{\infty}\left(p^{-1}(U)\right)$ to $C_{c}^{\infty}\left(p^{-1}(U)\right)$ and can be extended in a trivial way to an operator in $C^{\infty}(M)$. Such an operator will be called an elementary operator of class $\Psi^{m}(M / K)$ associated to the orbifold chart $\left(\tilde{U}, G_{U}, \phi_{U}\right)$.

By definition, the class $\Psi^{m}(M / K)$ consists of all operators $A$ in $C^{\infty}(M)$, which can be represented in the form

$$
A=\sum_{i=1}^{d} A_{i}+K,
$$

where $A_{i}$ is an elementary operator of class $\Psi^{*}(M / K)$ associated to an orbifold chart $\left(\tilde{U}_{i}, G_{U_{i}}, \phi_{U_{i}}\right)$ as in Proposition $4, i=1, \ldots, d$, and $K \in \Psi^{-\infty}(M)$.

Remark 3. When the group $K$ is discrete (and, therefore, finite), the algebra $\Psi^{*}(M / K)$ is the crossed product algebra $\Psi^{*}(X) \rtimes K$.

The principal symbol of the operator $\bar{A}$ given by (4) is a smooth function on $K \times K \times \tilde{U} \times$ $\left(\mathbb{R}^{n} \backslash\{0\}\right)$ given by

$$
\sigma(\bar{A})\left(k, k^{\prime}, y, \eta\right)=a_{m}\left(k, k^{\prime}, y, \eta\right), \quad k, k^{\prime} \in K, \quad y \in \tilde{U}, \quad \eta \in \mathbb{R}^{n} \backslash\{0\},
$$

where $a_{m}$ is the degree $m$ homogeneous component of the complete symbol $a$. This function is $G_{U}$-invariant and defines a smooth function on $\left(K \times K \times \tilde{U} \times\left(\mathbb{R}^{n} \backslash\{0\}\right)\right) / G_{U}$. We put

$$
\begin{aligned}
& \sigma(A)\left((k, y, \eta), k^{\prime}\right)=\sigma(\bar{A})\left(k,\left(k^{\prime}\right)^{-1} k, y, \eta\right), \\
& (k, y, \eta) \in\left(K \times \tilde{U} \times\left(\mathbb{R}^{n} \backslash\{0\}\right)\right) / G_{U}, \quad k^{\prime} \in K .
\end{aligned}
$$

Let $S^{m}\left(T_{K}^{*} M \rtimes K\right)$ be the space of all smooth functions on $\left(T_{K}^{*} M \backslash\{0\}\right) \rtimes K$ homogeneous of degree $m$ with respect to the $\mathbb{R}$-action given by the multiplication in the fibers of the vector bundle $\pi: T_{K}^{*} M \rightarrow M$. The principal symbol $\sigma(A)$ of an operator $A \in \Psi^{m}(M / K)$ given in local coordinates by the formula (5) is globally defined as an element of the space $S^{m}\left(T_{K}^{*} M \rtimes K\right)$.

One can introduce the structure of involutive algebra on $S^{*}\left(T_{K}^{*} M \rtimes K\right)$ (see (1) and (2)), and show that the principal symbol mapping

$$
\sigma: \Psi^{m}(M / K) \rightarrow S^{m}\left(T_{K}^{*} M \rtimes K\right)
$$

satisfies, for any $A \in \Psi^{m_{1}}(M / K)$ and $B \in \Psi^{m_{2}}(M / K)$,

$$
\sigma(A B)=\sigma(A) \sigma(B), \quad \sigma\left(A^{*}\right)=\sigma(A)^{*} .
$$

Example 1. For any $f \in C^{\infty}(M \times K)$, the operator $R(f)$ given by (3) belongs to $\Psi^{0}(M / K)$ and its principal symbol $\sigma(R(f)) \in S^{0}\left(T_{K}^{*} M \rtimes K\right)$ is given by

$$
\sigma(R(f))=\pi_{G}^{*} f
$$

where $\pi_{G}: T_{K}^{*} M \times K \rightarrow M \times K$ is the natural projection. 


\subsection{Noncommutative Egorov theorem}

As above, let $X$ be a compact orbifold and $X \cong M / K$ a quotient presentation for $X$. Assume that $P$ is an elliptic, first-order pseudodifferential operator on $X$ with real principal symbol $p \in S^{1}\left(T^{*} X\right) \cong S^{1}\left(T_{K}^{*} M\right)^{K}$. By Proposition 3, there exists an elliptic, first-order pseudodifferential operator $\tilde{P}$ with real principal symbol $\tilde{p} \in S^{1}\left(T^{*} M\right)^{K}$, which commutes with $K$ and whose restriction to $C^{\infty}(M)^{K}$ agrees with $P$. Denote by $\tilde{f}_{t}$ the Hamiltonian flow of $\tilde{p}$ on $T^{*} M \backslash\{0\}$. Recall that the flow $\tilde{f}_{t}$ preserves the conormal bundle $T_{K}^{*} M$, and its restriction to $T_{K}^{*} M$ commutes with the $K$-action on $T_{K}^{*} M$.

Define a flow $F_{t}$ on $T_{K}^{*} M \rtimes K$ by the formula

$$
F_{t}(\nu, k)=\left(\tilde{f}_{t}(\nu), k\right), \quad(\nu, k) \in T_{K}^{*} M \rtimes K .
$$

Observe that, due to $K$-invariance of the flow $\tilde{f}_{t}$, the induced map $F_{t}^{*}$ on $C^{\infty}\left(T_{K}^{*} M \rtimes K\right)$ is an involutive algebra automorphism. The one-parameter automorphism group $F_{t}^{*}$ of the algebra $C^{\infty}\left(T_{K}^{*} M \rtimes K\right)$ is called the noncommutative bicharacteristic flow of the operator $P$ (associated to the quotient presentation $X \cong M / K$ ).

Remark 4. One can show that the flow $F_{t}^{*}$ is Hamiltonian with respect to the natural noncommutative Poisson structure on the algebra $C^{\infty}\left(T_{K}^{*} M \rtimes K\right)$ and $p$ is the corresponding Hamiltonian (see [13] for more details).

The noncommutative version of the Egorov theorem for orbifolds reads as follows.

Theorem 3. For any $A \in \Psi^{m}(M / K)$ with the principal symbol $a \in S^{m}\left(T_{K}^{*} M \rtimes K\right)$, the operator

$$
\Phi_{t}(A)=e^{i t \tilde{P}} A e^{-i t \tilde{P}}
$$

is an operator of class $\Psi^{m}(M / K)$. Moreover, the principal symbol a $(t) \in S^{m}\left(T_{K}^{*} M \rtimes K\right)$ of $\Phi_{t}(A)$ is given by

$$
a(t)=F_{t}^{*}(a) .
$$

Proof. The $K$ orbits on $M$ define a foliation $\mathcal{F}$ on $M$ with compact leaves. The holonomy groupoid $G$ of this foliation coincides with the transformation groupoid $M \rtimes K$. The conormal bundle of $\mathcal{F}$ coincides with $T_{K}^{*} M$. As it is well-known in foliation theory, the conormal bundle of the foliation carries a natural foliation, $\mathcal{F}_{N}$, on $T_{K}^{*} M$, called the linearized or the lifted foliation. In our case, this foliation is given by the $K$ orbits on $T_{K}^{*} M$. The holonomy groupoid $G_{\mathcal{F}_{N}}$ of $\mathcal{F}_{N}$ is the transformation groupoid $T_{K}^{*} M \rtimes K$.

The algebra $\Psi^{*}(M / K)$ of noncommutative pseudodifferential operators associated with the quotient presentation $X \cong M / K$ is a particular case of the algebra $\Psi^{*,-\infty}(M, \mathcal{F})$ of transversal pseudodifferential operators on the compact foliated manifold $(M, \mathcal{F})$ introduced in [10]. In this setting, Theorem 3 is a straightforward consequence of the Egorov theorem for transversally elliptic operators proved in [11] (see also [12]).

Example 2. Suppose that $X$ is a compact manifold (considered as an orbifold). Then a quotient presentation for $X$ is just a $K$-principal bundle $\phi: M \rightarrow X$. Let $g_{X}$ be a Riemannian metric on $X$. Choose a bi-invariant metric on $K$ and a connection on the principal bundle $\phi: M \rightarrow X$. There exists a unique $K$-invariant metric on $X$, which makes the map $\phi:\left(M, g_{M}\right) \rightarrow\left(X, g_{X}\right)$ into a Riemannian submersion, with the fibers isometric to $K$. Such a metric is sometimes called the Kaluza-Klein metric of the connection.

Then, for a Hamiltonian $H \in C^{\infty}\left(T^{*} X \backslash\{0\}\right)$ given by

$$
H(x, \xi)=|\xi|_{g_{X}}, \quad(x, \xi) \in T^{*} X,
$$


the Hamiltonian flow $\tilde{f}_{t}$ on $T^{*} M$ is the geodesic flow of the metric $g_{M}$, and the reduced Hamiltonian flow $f_{t}$ on $T^{*} X$ is the geodesic flow of the metric $g_{X}$.

The corresponding quantum dynamics on $L^{2}(X)$ and $L^{2}(M)$ are described by the operators $P=\sqrt{\Delta_{X}}$ and $\tilde{P}=\sqrt{\Delta_{M}}$ respectively, where $\Delta_{X}$ and $\Delta_{M}$ are the Laplacians of the metrics $g_{X}$ and $g_{M}$ respectively. It is well known that the operator $\Delta_{M}$ can be expressed in terms of Bochner Laplacians acting on sections of vector bundles over $X$ associated with the principal bundle $\phi: M \rightarrow X$.

In the case when $K=O(n)$ and $M$ is the orthonormal frame bundle $F(X)$ of $X$, the restriction of the geodesic flow $\tilde{f}_{t}$ to $T_{K}^{*} M$ is closely related with the frame flow on the frame bundle $F(X)$ on $X$ (see [12] for more details).

Remark 5. In this case, both classical and quantum dynamical systems are noncommutative. It would be interesting to extend some basic results on quantum ergodicity to this setting. For instance, one can introduce the notion of ergodicity for the bicharacteristic flow $F_{t}^{*}$ on the noncommutative algebra $C^{\infty}\left(T_{K}^{*} M \rtimes K\right)$ and compare this notion with an appropriate notion of quantum ergodicity for the operator $P$ itself.

\section{Acknowledgements}

The author was partially supported by the Russian Foundation of Basic Research (grant no. 10-01-00088).

\section{References}

[1] Adem A., Leida J., Ruan Y., Orbifolds and stringy topology, Cambridge Tracts in Mathematics, Vol. 171, Cambridge University Press, Cambridge, 2007.

[2] Bucicovschi B., Seeley's theory of pseudodifferential operators on orbifolds, math.DG/9912228.

[3] Dryden E.B., Gordon C.S., Greenwald S.J., Webb D.L., Asymptotic expansion of the heat kernel for orbifolds, Michigan Math. J. 56 (2008), 205-238.

[4] Girbau J., Nicolau M., Pseudodifferential operators on $V$-manifolds and foliations. I, Collect. Math. 30 (1979), 247-265.

[5] Girbau J., Nicolau M., Pseudodifferential operators on V-manifolds and foliations. II, Collect. Math. 31 (1980), 63-95.

[6] Guillemin V., Uribe A., Wang Z., Geodesics on weighted projective spaces, Ann. Global Anal. Geom. 36 (2009), 205-220, arXiv:0805.1003.

[7] Jakobson D., Strohmaier A., High energy limits of Laplace-type and Dirac-type eigenfunctions and frame flows, Comm. Math. Phys. 270 (2007), 813-833, math.SP/0607616.

[8] Kawasaki T., The signature theorem for $V$-manifolds, Topology 17 (1978), 75-83.

[9] Kawasaki T., The index of elliptic operators over V-manifolds, Nagoya Math. J. 84 (1981), 135-157.

[10] Kordyukov Yu.A., Noncommutative spectral geometry of Riemannian foliations, Manuscripta Math. 94 (1997), 45-73.

[11] Kordyukov Yu.A., Egorov's theorem for transversally elliptic operators on foliated manifolds and noncommutative geodesic flow, Math. Phys. Anal. Geom. 8 (2005), 97-119, math.DG/0407435.

[12] Kordyukov Yu.A., The Egorov theorem for transverse Dirac-type operators on foliated manifolds, J. Geom. Phys. 57 (2007), 2345-2364, arXiv:0708.1660.

[13] Kordyukov Yu.A., Noncommutative Hamiltonian dynamics on foliated manifolds, J. Fixed Point Theory Appl. 7 (2010), 385-399, arXiv:0912.1923.

[14] Marsden J., Weinstein A., Reduction of symplectic manifolds with symmetry, Rep. Math. Phys. 5 (1974), 121-130.

[15] Moerdijk I., Mrčun J., Introduction to foliations and Lie groupoids, Cambridge Studies in Advanced Mathematics, Vol. 91, Cambridge University Press, Cambridge, 2003.

[16] Sjamaar R., Lerman E., Stratified symplectic spaces and reduction, Ann. of Math. (2) 134 (1991), 375-422.

[17] Stanhope E., Uribe A., The spectral function of a Riemannian orbifold, Ann. Global Anal. Geom. 40 (2011), 47-65. 